\title{
Comprehensive Diabetes and Non-Communicable Disease Educator in the Low-Resource Settings
}

\author{
Madhur Dev Bhattarai ${ }^{1}$ \\ 'Nepal Diabetes Association, Kathmandu, Nepal.
}

\begin{abstract}
The role of self-management education in diabetes and other major non-communicable diseases is clearly evident. To take care of and educate people with diabetes and other major NCD under the supervision of medical professionals and for education of other health care professionals, Comprehensive Diabetes and NCD Educators are needed in the routine service in peripheral health clinics and hospitals. The areas of training of CDNCD educator should match with the cost-effective interventions for diabetes and other major NCD that are feasible and planned for implementation in primary care in the low resource settings. Most of such interventions are part of diabetes education as required for Diabetes Self-Management Education programmes and traditional Diabetes Educator. The addition of use of inhaled steroids and bronchodilator in chronic respiratory disease and identification of presenting features of cancer, also required for many people with diabetes with various such common co-morbidities, will complete the areas of training of traditional Diabetes Educator as that of CDNCD Educator. Staff nurse and health assistants, who are as such already providing routine clinical service to all patients including with diabetes and major NCD in peripheral health clinics and hospitals, are most appropriate for CDNCD Educator training. The training of CDNCD Educator, like that of traditional Diabetes Educator, requires fulfilment of sufficient hours of practical work experience under supervision and achievement of the essential competencies entailing at least 6 month or more of intensive training schedules to be eligible to appear in its final certifying examination.
\end{abstract}

Keywords: CDNCD educator; diabetes; diabetes educator; DSME; global NCD alliance; NCD; noncommunicable diseases; WHO PEN.

\section{INTRODUCTION}

Diabetes education is critical for effective selfmanagement. ${ }^{1-4}$ The history of modern structured Diabetes Self-Management Education (DSME) programmes for the patient began in Germany with a residential 1-week course. ${ }^{5}$ Now such programmes are mostly non-residential. There are different Diabetes SelfManagement Education programmes generally with 6 to 16 people, in some 20 to 24 including family members, from 6 hour to a few days divided in various sessions with or without 1 to 3 follow-up sessions for persons with Type 1 and Type 2 and/or for those at increased risk of developing type 2 diabetes programmes. ${ }^{4-6}$
Other direct and online Educational programme for people with diabetes are also run by different national and international organizations and associations in the world. ${ }^{2,7}$

In the industrialized countries, the nurses and others are trained as Diabetes Educator to provide routine service to the patients along with the medical professionals in the hospitals and general practice and to carry out

Correspondence: Dr. Madhur Dev Bhattarai, Nepal Diabetes Association, Kathmandu, Nepal. Email: mdb@ntc.net. np, Phone no.: 977-1-4434281. 
different educational programmes in the community. ${ }^{3,8}$ Diabetes is a chronic life-long disease with many potential complications and the care and education have to cover management of hypertension, hyperlipidaemia, cardiovascular diseases (CVD) and other co-morbidities. Reduction of cardiovascular and other complications is the focus of comprehensive management in diabetes. ${ }^{1}$ The article will briefly cover the areas, levels, and outline of the training of educator for diabetes and major non-communicable diseases (NCD) relevant to the low-resource settings.

\section{Areas of training for educators}

The four major NCD - CVD (heart disease and stroke), cancer, chronic respiratory disease and diabetes together are responsible for 28 million deaths a year and make the largest contribution to the NCD burden in lowand middle-income countries. ${ }^{9}$ Nearly $80 \%$ of deaths due to CVD occur in low- and middle-income countries. ${ }^{10}$ The four major NCD have many common risk factors like physical inactivity, unhealthy diet, overweight and obesity, smoking, alcohol, hypertension, poor mother and child health care and others. ${ }^{11}$ The WHO Package of Essential Noncommunicable (WHO PEN) Disease Interventions for primary care is an innovative and action-oriented response to the above challenges with cost-effective interventions that are feasible for implementation even in low-resource settings. ${ }^{9}$ The interventions are for detection, prevention, treatment and care of CVD and its risk factors, diabetes, chronic respiratory disease (asthma and chronic obstructive pulmonary diseases - COPD) and cancer which can be delivered by primary care physicians and nonphysician health workers in primary care. ${ }^{9}$ Most of such interventions are also part of education useful for Diabetes Self-Management Education and for traditional Diabetes Educator. The emergency and regular health check-ups and management, including the required antibiotic use and vaccination, are also needed for people with diabetes. The only two additional areas remaining to be included to match the training of health workers with the health service as per the need of the people are the appropriate use of inhaled bronchodilator and steroids in chronic respiratory diseases and the identification of presenting features of cancer to refer to next level for confirmation of diagnosis and management.

Chronic respiratory disease and pulmonary infections including tuberculosis are common problems in the community as well as among people with diabetes and the knowledge of its management will be added benefit to the Diabetes Educators and their patients. Almost one-fourth of total lost output from NCD in low- and middle-income countries is due to respiratory diseases. ${ }^{10}$ The correct technique and use of metereddose inhaler and dry power inhaler also needs education, motivation and monitoring of patients as required for smoking cessation, alcohol control, and adherence to the healthy lifestyle and medication for diabetes, blood pressure and other CVD. Similarly, people with diabetes also have an increased risk of cancer mortality and epidemiologic evidence suggests that cancer incidence is associated with diabetes as well as certain diabetes risk factors and diabetes treatments. ${ }^{12,13}$ The addition of use of bronchodilator and inhaled steroids in chronic respiratory disease and identification of presenting features of cancer covered in the WHO $\mathrm{PEN}^{9}$ will complete the areas of training of traditional Diabetes Educator as that of Comprehensive Diabetes and NCD (CDNCD) Educator (Figure 1) particularly useful in lowresource setting facilitating their integration in the local Peripheral Health Service system. The concept of CDNCD Educator is also in line with the principle of global NCD Alliance founded in 2009 by the four non-governmental organization federations of diabetes, tuberculosis and lung disease, cancer, and heart. ${ }^{14}$ It would, thus, helps to consolidate all the available resources for the low-resource settings. Such CDNCD Educator could work in peripheral health service and hospitals under the supervision of medical professionals with the help of available resources, evidence based clinical protocols and flow chart with referral criteria. They can be given any further relevant skill training for the technologies and tools whenever available and as required for them in their working place; a few examples of such technologies when resources permit are given in the WHO $\mathrm{PEN}^{9}$. The training of CDNCD Educator in this way will strengthen both Peripheral Health Service (PHS) in the network of Rural and Urban Health Centers and rural District Hospitals and Specialist Health Services (SHS) in public hospitals and Medical Teaching Institutions. It will also help different Vertical Public Health Programme Services (VPHPS) to come together to share and effectively utilize their resources. The PHS, SHS and VPHPS are three of the five fundamental health service responsibility of any Government to provide the base health service to the people. ${ }^{15}$

Levels of education and training of health care workers in diabetes and major NCD

Health care settings are managed by medical and health care professionals with the support of other complementary workers. The patients require accurate educational information and follow-up sessions by competent professionals to self-manage safely, however physicians often have only time to address the immediate problem during a patient visit. ${ }^{16}$ The doctors usually have to deal with other more immediate 
problems of the patients. ${ }^{17}$ All health care workers need to be prepared for the different situations depending on their responsibilities and workplace where patients interact with them. In order for the members of staff to carry out their roles effectively, no matter the level of their career, the knowledge and skills standards have to be set for everyone who is in regular contact with patients with diabetes ${ }^{8}$ and other major NCDs. Broadly the levels of education and training in diabetes and other major NCD for health care professionals and other health staffs can be divided into four groups (Figure 2) to plan and manage such programmes accordingly in the low resource settings.

General and additional related education and training in diabetes and other major NCD to the health care professionals (Number 1 and 2 areas in the Figure 2) can be managed by the short term education and trainings of a few days by the concerned units and educators in the institutions with or without support of other organizations. The need of appropriate planning and management of training of health care professionals as CDNCD Educator (Number 3 in Figure 2) for the routine service in peripheral health system and hospitals to take care of the patients with diabetes and other major NCD under the supervision of medical professionals is obvious in the low resource settings. To ensure people receive high-quality care, organizational changes may also need to be considered in the health care settings, ${ }^{18}$ e.g. by incorporating the job of CDNCD Educator and their career. Such cadre of CDNCD educator providing regular service in peripheral health system and hospitals can be easily trained further to manage specific education programme of diabetes and other major NCDs (Number 4 in Figure 2) in different places and patient populations in the community as well as for other health care professionals (number 1 and 2 in Figure 2). Without such cadre of CDNCD educator, it may not be possible to conduct the effective selfmanagement education programme for the patients regularly either in the routine health care settings or in the community. Thus training to prepare this key group of CDNCD Educator requires particular attention and is discussed further below.

\section{Outline of CDNCD Educator training}

Personnel for training: Nurses have been utilized most often as instructors in the delivery of formal Diabetes Self Management Education programme with the support of dieticians and other Health Care Professionals, apart from their involvement in the education and care of patients in the routine health care as Diabetes Specialist Nurse. ${ }^{3,8,16}$ Systematic reviews report the best results in terms of improved metabolic control, weight loss, and diabetes-related knowledge in cases where mainly nurses provide patient education combined with structured and regular patient review. ${ }^{19}$ To achieve Certified Diabetes Educators (CDE) credentials in the US, the Eligible health care professionals include nurses and others as appropriate to the local situation with a minimum of 2 years of their practice experience. ${ }^{20}$ Staff nurse and health assistant with at least tenplus-two (certificate) level of education in the health profession as such already providing routine clinical service to all the patients including with diabetes and major NCD in peripheral health clinics and hospitals under the supervision of medical professionals are most appropriate for CDNCD Educator training in the low resource settings.

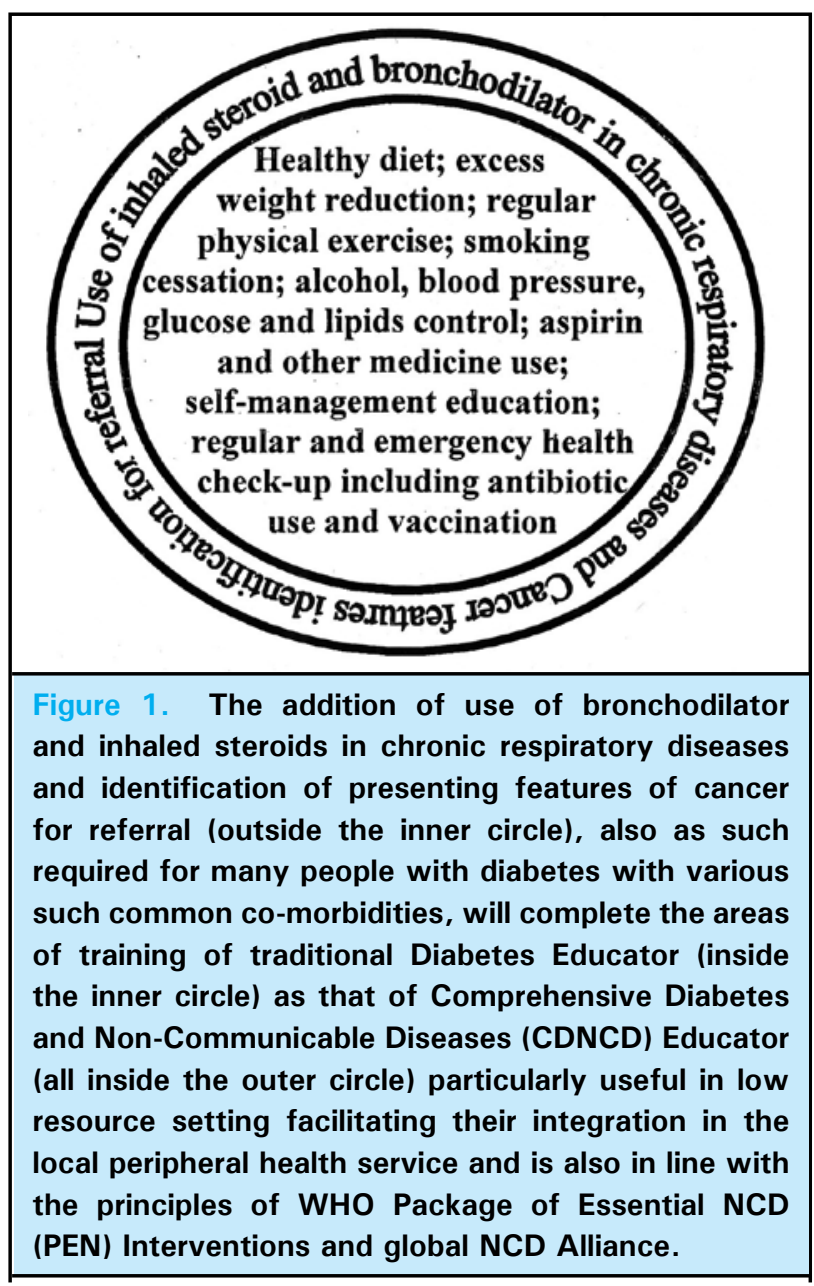

Training principle, duration, and material: For any education and training there are various learning theories and approaches, viz. behaviorism, cognitivism, social cognitive, constructivism, reflection, self-directed learning, humanistic approach, experiential, active learning, older and new or cognitive apprenticeship system and many others with increasing emphasis on structured training focusing on formal skills training, evidence-based accountability, objective evaluation, 
and competence-based assessment. ${ }^{21,22}$ Such theories and approaches of learning are incorporated in various forms in the structured residential training of medical professionals. The concept of the structured training programme of medical residency with actual working in the field under the supervision of Faculty with various teaching, learning and communication activities applies to any similar training of other Health Care Professionals, including that of CDNCD Educator. The criteria of certification of such training indicate the quality of the programme prospectively, i.e. fitness to practice in the concerned job later by the trainees, reflecting the principle of "Fitness for purpose" 23 of achievement of the purpose of the assessment plan. The certification of the fitness of the trainees to practice in their field is assured by the fulfillment of the objective training criteria, viz the entry criteria to join the concerned training and the eligibility criteria for the certification process with exit examination as an example in the low resource settings, ${ }^{24}$ while actually performing the job in a graded manner under the supervision of Faculty so that they can later perform the job independently.

\begin{tabular}{|l}
\hline Further training to run specific \\
self management education \\
programmes for different groups \\
of people with diabetes and other \\
major NCD \\
To train as Comprehensive Diabetes and Non-Communicable Diseases \\
(CDNCD) Educator for the routine service in peripheral health system and \\
hospitals to take care of the people with diabetes and other major NCD \\
under the supervision of medical professionals \\
for the specific \\
unit
\end{tabular}

To be eligible for the certification examination of Diabetes Educators in the US, the healthcare professional should have 1000 hours of practice experience in diabetes self-management education along with a minimum of 15 hours of continuing education activities in diabetes in recent years. ${ }^{20}$ For academic session, a unit of credit equates to three hours of students per week, e.g. 1 hour lecture plus 2 hours of home work or 3 hours of lab for 16 weeks. ${ }^{25}$ Fulfillment of such hours of experience and teaching and learning activities entails at least 6 month or more of intensive training schedules. The number of candidates to be trained would depend on the workload in the training unit and feasibility of conducting small group sessions and discussion for effective learning and fulfillment of the training criteria. The trainees would work under the supervision of the physicians providing care and education to the patients and completing the training requirements, the overview of which are outlined by the planning and printed materials required for the training in Table 1 and the logbook details in Table 2 for documentation of the fulfillment of the eligibility criteria to appear in its final certifying examination. The assessment in the certifying examination is based on the areas and criteria of the training conducted.

Topics and modules for teaching and discussion: Selfmanagement education programmes incorporating behavioral and psychosocial strategies with a comprehensive plan of care demonstrate improved outcomes in diabetes ${ }^{3}$ with its various complications of the major NCD. Healthy eating, being active, monitoring, taking medications, problem solving, healthy coping, reducing risks and others are the self-care behviours of people with diabetes ${ }^{28}$ and other major NCDs. Similarly assessment (of the individualized patients), goal setting, planning, implementation, evaluation and documentation are the required standards of practice for their educators. ${ }^{28}$ While counseling and goal setting, the 
experience, with documentation in the logbook (Table 2), of managing people with various complications like renal failure, visual loss, coronary health disease, strokes, diabetic foot and others will help the trainers to motivate the patients to adhere to healthy lifestyles and prescribed medications to control BP and other risk factors. The goal setting should be discussed with the patients and planned for different required and possible dietary, physical activity, and therapeutic factors along with follow-up dates. The teaching and learning sessions on principle and method of communication, counseling and motivation with interactive sessions will further help to acquire such skills.

Patho-physiology, epidemiology, and clinical guidelines; culturally-competent supportive care across the lifespan; teaching and learning; self-management education; and program and business management are the five domains of the competencies for the educators of diabetes ${ }^{29}$ which are also applicable to other major NCD. Such areas and various aspects of diabetes and other major NCD particularly from the perspectives of management of patients as per the guidance of medical professionals and evidence-based clinical protocols and flow charts should be covered by different types of sessions. Salient practical aspects of management of diabetes and major NCD useful for the training of CDNCD Educators in the low resource settings are given in Table 3. The WHO PEN documents can be referred to for the examples of core set of evidence based interventions for reducing morbidity and mortality from major NCDs that are feasible for implementation in primary care in low resource settings as well as for the information on their cost effectiveness. ${ }^{9}$ For the use of education of healthcare professionals anywhere in the world, International Diabetes Federation has made available more than 800 slides of PowerPoint presentations with detailed speaker notes in different modules intended to be used in conjunction with the International Curriculum for Diabetes Health Professional Education containing extensive objectives, suggestions for faculty, activities and an evaluation process for each module along with some sample case studies and sample group-work activities $^{1,2}$ Similar materials and guidelines are available on line for diabetes and other major NCD management from different organizations, ${ }^{38,45-52}$ which and others, along with the national ones, can be appropriately referred to for the training.

\section{THE WAY FORWARD}

The need of the CDNCD Educator is evident for the routine service of patients in the peripheral health clinics and hospitals to care the patients with diabetes and other major NCD under the supervision of medical professionals and for education of other health care professionals and health staffs and of the people with diabetes and other major NCD and general public in the community. The areas of the training should match with the cost-effective interventions for diabetes and other major NCD that are feasible and planned for implementation in primary health care even in the low-resource settings. Most of such interventions are as such part of diabetes education as needed for the Diabetes Self-Management Education programmes and traditional Diabetes Educator. The addition of use of bronchodilator and inhaled steroids in chronic respiratory disease and identification of presenting features of cancer will complete the areas of training of traditional Diabetes Educator, with added benefit to them and their patients with various such common complications and co-morbidities, as that of CDNCD Educator. The training of the CDNCD Educator, as that of traditional Diabetes Educator, requires fulfillment of sufficient hours of practical work experience under supervision and the achievement of the essential competencies, which entail at least 6 month or more of intensive training schedules to be eligible to appear in its final certification examination. Various existing national and international practices, evidences, guidelines and materials of the training of medical, health care professionals, and diabetes educators, and of the management of the major NCD, as discussed in the article, guide the areas, principles, duration and other requirements and details of CDNCD Educator training. The training of the CDNCD Educator in this way and their incorporation in the health system will help the Peripheral Health Service, Specialist Health Service, and Vertical Public Health Programme Service, the three fundamental health service responsibility of any Government, to achieve their primary goal of providing the basic health service to the people. 
Table 1. Planning and printed materials required for the training of Comprehensive Diabetes and Non-Communicable Disease (CDNCD) Educator in the low resource settings.

1. Logbook (Table 2) for documentation of the fulfillment of the eligibility criteria to appear in the final certifying examination.

2. Schedules of lecture and discussion sessions

3. Schedules of assignments and assessments

4. Handy flip chart as the printed folders and/or in the electronic forms ${ }^{*}$ for trainees to carry to explain patients about pathophysiology, symptoms, signs and complications of each of the major NCD; diet, carbohydrate counting, physical activity, weight chart as per the recommended body mass index for the population, and waist-hip measurement and chart; steps, checklist $t$, and required information for different procedures; step care management of major NCD; flow-chart with treatment guidelines and referral criteria; and summary points of regular investigations, treatments and prevention of various major NCD

5. Manual, booklets, flowcharts and guidelines $\ddagger$ based on various national and international ones about the major NCD for the trainees to study and prepare for assessments

6. Certificate $\S$ with documented number of hours of practical work experience under supervision; case histories completed; procedure explanations and counseling recorded; lectures, discussions and group sessions attended; and assignments and assessments cleared.

Note: The electronic forms of handy flip charts, flow chart and guidelines can be made available for the low resource settings by the national and international agencies.

*Hand-outs for people and patients may be prepared from such flip charts

$t$ The provision of 21 predefined educational areas of syringe and insulin pen routes of insulin administration during simulations ${ }^{26}$ is a useful example of checklist.

¥ The guidelines may be regularly updated and made available in the website by the institutions providing such training or the national bodies for the use of trainee and already trained educators.

$\S$ Such training course should ideally be accredited by the state or national official vocational training body or professional body counsel.

Table 2. Logbook details for documentations of the fulfillment of the eligibility criteria to appear in the final certifying examination of the training of Comprehensive Diabetes and Non-Communicable Disease (CDNCD) Educator in the low resource settings

1. Attendance recording with hours of actual working as educator under supervision in the concerned specialties

2. Case history recordings: Pages for case history recording (with subheadings to write clinical history and physical findings; physical, psychological and financial effects and difficulties due to the disease, complications and/or the treatments to the patients and their family; along with goal setting with the patient for different plans of management) of at least one hundred people of diabetes including some with renal failure, stroke, myocardial infarction, laser eye treatment, pregnancy, breastfeeding, and type 1 diabetes as well as of chronic respiratory diseases like chronic obstructive pulmonary diseases and asthma and cancers.

3. Procedure explanation and counseling recordings: Pages for common day-to-day procedure explanations and counseling recordings while educating the patients (with subheadings to write difficulties, result, correlation with other factors like food or medications or other results, learning of the trainee, and others) in general twenty to fifty* each of monofilament, glucometer and peak expiratory flow meter examinations along with interpretation of the results; insulin injection; fundal camera procedures; step-care management in chronic respiratory diseases; use of meter-dose inhaler with or without spacer, dry powder inhaler, vascular risk prediction charts; counseling with mothers for appropriate nutrition, promotion of exclusive breast feeding for six months, gestational diabetes management, and post-partum diet and weight; and others.

4. Attendance recording of lectures and study topic discussions

5. Attendance recording of group discussions with trainees and group discussions with patients

6. Assignments and assessment sessions completed and cleared

Note: The case histories, procedures and other practical experience should be discussed along with learning point regularly almost every day with the group of trainees by Faculty members.

*The numbers of procedures required while teaching the patients will depend on previous experience, procedure complexity, and competency required, e.g. observing, assisting, performing under supervision, and performing independently. ${ }^{27}$ 
Table 3. Salient practical aspects of management of diabetes and major non-communicable diseases (NCD)* useful for the training of Comprehensive Diabetes and NCD (CDNCD) Educator in the low resource settings

Glycemic control of

Type 2 diabetes : Initial stage

\section{Glycemic control} of

type 2 diabetes : Later stage/s

Glycemic control of

type 1 diabetes

High blood pressure (BP)

Cardiovascular disease (CVD) prevention and management

Chronic obstructive airway diseases

Early detection of cancer

Prevention of cancer
- Increasing physical activity and even resistance training exercise ${ }^{30} \dagger$

- Minimization of refined sugars and soft drinks with adequate water intake

- Reducing excess weight as per recommended BMI for the population - lower the better $\S, 0, \emptyset$

- Gradual building-up of dose of metformin to the tolerable recommended level* *

- As required temporary use of sulfonylurea** or insulin for complications, infections, hyperglycemia during immobility or initial building-up of metformin dose or other reasons

Note: A few patients may not respond to metformin or even sulfonylurea from the beginning

- Maintaining tolerable recommended dose of metformin**

- As required temporary use of sulfonylurea** or insulin for hyperglycemia due to any reason

- Choice of next drug/s considering glucose control; frequency of monitoring required; risk of hypoglycemia; other side-effects, safety profile and cost of drug/s; comorbidities; guidelines; and mobility, life style and preference of the patient $\dagger \dagger, \ddagger \ddagger, \S \S$

- Insulin remains effective for hyperglycemia in any situation in diabetes $\ddagger \ddagger, \S \S$

Flexible intensive insulin therapy to optimize glycemic control through independent selfmanagement including glucose monitoring, calculation of the meal-time fast-acting insulin dose, carbohydrate counting, sick-day rules, and others

- Responsible for about $50 \%$ of deaths due to CVD and for significant burden of kidney failure $^{10}$ and retinopathy

- Recommended salt intake $\square$

- Optimum level of BP as recommended for patients with or without diabetes, proteinuria, CVD, and others

- Controlling BP is most important and uncontrolled BP may be equivalent to untreated BP.

- Drug choice is as per co-morbidities, complications, cost, and recommendation; however, in most cases, more than one medicine is required $₫ \uparrow$

- Tobacco cessation*** and at least half-an-hour regular physical activity, e.g. brisk walking, at least 5 days a week, or equivalent cardio-respiratory exercise. ${ }^{30} \dagger$

- Prophylaxis of rheumatic fever

- Treatment of high BP, glucose and lipid and use of aspirin, statins, and other drug

- Avoid smoking and organophosphate and carbamate pesticide exposure

- Stepwise management with inhaled steroids and bronchodilators with correct technique of inhalers and spacer use

- Short term use of oral or systemic steroids as required for exacerbation of asthma

Unexplained bleeding; persistent unexplained symptoms; non-healing ulcer; increasing nodes; routine breast, cervix, and complete skin examination; and other recommendations for referral

Recommended vegetable and fruit intake and body weight, avoidance of tobacco*** and alcohol $\dagger \dagger \dagger$ use, practice of safer sex e.g. condom use, appropriate vaccinations and others

* Applicable to all the people with major NCD and the general population

- Recommended fruit, vegetable, calorie, fat (with adequate proportion of mono- and poly-unsaturated and saturated fatty acid), salt and other dietary intake; trans-fatty acid, tobacco and alcohol control; regular physical activity; and adequate water intake.

- Regular and emergency check-ups and management including appropriate vaccination, required antibiotic use and adherence to the recommended medical advice.

$\dagger$ Physical activities and exercise to be gradually buildup with necessary medical consultation. ${ }^{30}$ Walking is promoted wherever possible, e.g. 'morning-walk', 'working-walk', 'leisure-walk', 'walking inside house' etc. Resources and regulations are required for promotion of walking and physical activity in the community. ${ }^{31}$ 
Pedestrians, cyclists and public transport passengers are the top three hierarchy groups recommended while developing transport and traffic strategies. ${ }^{32}$

¥ Inadequate water drinking may lead to unnecessary intake of other energy-yielding beverage, alcohol, and food. Up to $81 \%$ of total water intake may thus unnecessarily be derived from beverages. ${ }^{33}$

§ Frequency of meal may have to be maintained or even increased if insulin, sulfonylurea or meglitinides is used to match the hypoglycemia effect and maximum action time of the medication and that may not be per se necessary for metformin or other currently available anti-hyperglycemic medicines used alone.

प All types of oil and fat have high calorie content. In many cultures in low resource settings excessive oil used in the cooking adds to the obesity, so promotion of healthy cooking at home and opening of healthy cooking society in the community for the healthy, tasty and innovative recipes may also be considered. ${ }^{31}$

I Apart from for primary prevention of type 2 diabetes, maintenance of body weight towards the mid- or even lower-body mass index (BMI) recommended for the population is also advocated before pregnancy, including as a programme to help control the diabetes epidemic, ${ }^{31}$ for optimum pre-pregnancy weight for prevention of gestational diabetes and maternal subclinical hyperglycemia to reduce the risk of immediate maternal and neonatal complications as well as of long-term possible metabolic effect on offspring

* * Fixed dose combination tablet of metformin, having long-term safety profile, with hypoglycemic drug sulfonylurea is irrational and will not allow gradual increment and maintenance of dose of metformin to the optimum level and continued effort to reduce sulfonylurea dose to the lowest possible level as the 'dynamic dose management of hypoglycemic medications like sulfonylurea or insulin' to the lowest possible dose.

$\dagger \dagger$ With gradual build-up, moderate dose of acarbose, having diabetes prevention activities ${ }^{34}$ and longsafety profile, may also be tolerated concomitantly with metformin ${ }^{35-37}$ like rest of other anti-diabetic medications $^{38}$. The long-term safety profile of such other drugs for possible use 'between metformin and acarbose to insulin' is currently not fully known and they are used by the physicians considering various factors.

$\ddagger \ddagger$ Apart from the overt manifestation of hypoglycemia, use of sulfonylurea or insulin often entails increased hunger and frequency of meals, frequent monitoring of glucose, difficulty in losing weight and even some weight gain. Maximum action time of the drugs should be considered while using any particular type and schedule of short- or long-acting sulfonylurea or insulin and while monitoring the blood glucose.

$\S \S$ Reduction of cardiovascular and other complications is the focus of comprehensive management in diabetes. Glycemic targets of glucose lowering therapies have to be individualized based on different elements of decision making like patient attitude and treatment efforts, risks potentially associated with hypoglycemia, disease duration, life expectancy, important comorbidities, established vascular complications, and resources, support system. ${ }^{39,40}$

므 Up to $80 \%$ of dietary salt may be unknowingly derived from salt added in the processing of different food and drink, ${ }^{41}$ e.g. baking powder, soy sauce, enriched wheat flour, sausage, tomato catsup bottled, cheese, butter, cookies, canned food-product, margarine, salty tea and others.

I Fixed dose combination tablet of BP drugs decreases pill burden.

*** Harmful tobacco products also include smokelesstobacco like snuff, gutkha, gul, chimo, mawa, nass, pan masala, tambaku and others. ${ }^{42}$ Smokers who quit smoking abruptly ('Cold Turkey' method) are more likely to be successful than those who quit gradually. ${ }^{43}$ After stopping by whichever way, tobacco use or smoking should not be done again even once; otherwise the habit may be resumed.

†† Light to moderate drinking is also associated with some increased risk of cancer, particularly in women and ex- and current-smokers. ${ }^{44}$ There is no medical reason to recommend alcohol intake. For CVD prevention, cessation of tobacco use, recommended physical activity and diet and control of BP and other risk factors are essential.

\section{REFERENCES}

1. Consultative Section on Diabetes Education. International Curriculum for Diabetes Health Professional Education of IDF. Brussels: International Diabetes Federation, 2008

2. International Diabetes Federation. Diabetes Education Modules. Brussels: International Diabetes Federtion, 2011.
3. Funnell MM, Brown TL, Childs BP, et al. National Standards for Diabetes Self-Management Education. Diabetes Care 2007;30(6):1630-7.

4. Clarke A. Delivering diabetes education in the community to meet local needs. J Diabet Nursing 2008;12(9):348-57. 
5. Mansell P. The Dose Adjustment for Normal Eating (DAFNE) education Programme. J Diabet Nursing 2012;16(9):364-9.

6. Forde R, Dinneen S, Humphreys M, et al. Review of Diabetes Structured Education. Dublin: Republic of Ireland, 2009.

7. Blonde L, Warren-Boulton E. Translating science into practice: the US National Diabetes Education Program. DiabetesVoice 2007;52(1): 20-23

8. Walsh N, George S, Priest L, et al. The current status of diabetes professional educational standards and competencies in the UK - a Position Statement from the Diabetes UK Healthcare Professional Education Competency Framework Task and Finish Group. Diabetic Medicine 2011;28:1501-7.

9. WHO. Package of Essential Non-communicable Disease Interventions (WHO PEN) for Primary Care in Low-Resource Settings. Geneva: WHO, 2010.

10. WHO. A Global Brief on Hypertension - Silent Killer, Global Public Health Crisis. Genva: WHO, 2013.

11. WHO. World health report 2002: Reducing risks, promoting healthy life. Geneva: WHO, 2003.

12. Giovannucci E, Harlan DM, Archer MC, et al. Diabetes and cancer: a consensus report. Diabetes Care 2010; 33(7):1674-85.

13. Emerging Risk Factors Collaboration, Seshasai SR, Kaptoge $\mathrm{S}$, et al. Diabetes mellitus, fasting glucose, and risk of cause-specific death. N Engl J Med 2011; 364(9):829-41.

14. NCD Alliance. Non-Communicable Diseases: Join the Fight - An Online Advocacy Toolkit. NCD Alliance, 2013.

15. Bhattarai MD. Facilitation of free residential training inside the country - The fundamental health service responsibility of the Government and its regulatory body. J Nepal Med Assoc 2015;53(197):40-69.

16. Moshang J. Getting the job done: the diabetes nurse specialist. Int J Clin Pract 2007;61(9):1429-31.

17. Pishdad GR, Pishdad R, Pishdad P. A nurse-managed diabetes care programme. Int J Clin Prac 2007;61(9):1492-7

18. Gosden CA, Barnard K, Williams DRR, Tinati T, Turner B, Holt RIG. A decade in diabetes specialist services, 2000 to 2011, in England: the views of consultant diabetologists and diabetes specialist nurses amidst persistent healthcare delivery change. Dabetic Medicine 2015;32:1662-6.

19. Korsatko S, Habacher W, Rakovac I, et al. Evaluation of a Teaching and Treatment Program in Over 4,000 Type 2 Diabetic Patients After Introduction of Reimbursement Policy for Physicians. Diabetes Care 2007;30(6):1584-6.

20. National Certification Board of Diabetes Educator. Certification Examination for Diabetes Educators. St. Olathe (US): NCBDE, 2014.

21. Bleakley A, Bligh J, Browne J. Medical Education for the Future: Identity, Power and Location. London: Springer, 2011.

22. Kaufman DM, Mann KV, Jennet PA. Teaching and Learning in Medical Education: How Theory can Inform Practice. Edinburgh: Association for the Study of Medical Education (ASME); 2000.
23. Harvey L, Green D. Defining quality. Assess Eval High Educ 1993;18:9-34

24. Bhattarai MD. Twenty Lessons to Incorporate EBM Concept and Practices into Medical Education. In: Sitaras NM, Ed. Evidence Based Medicine (EBM) - Closer to Patients or Scientists? Rijeka: INTECH, 2012: 29-54.

25. Los Angeles Southwest College. Definition of "Credit hour" - The Carnegie Unit: How to Calculate Student Contact Hours. Los Angeles: LASC, 2015.

26. Taylor CG, Bynoe K, Worme A, et al. A checklist that enhances the provision of education during insulin initiation simulation: a randomized controlled trial. Diabet Med. 2015 Sep 4. doi: 10.1111/dme.12956. [Epub ahead of print].

27. Tariq M, Bhulani N, Jafferani A, et al. Optimum number of procedures required to achieve procedural skills competency in internal medicine residents. BMC Medical Education, 2015;15:179.

28. American Association of Diabetes Educators. The Scope of Practice, Standards of Practice, and Standards of Professional Performance for Diabetes Educator. Chicago: American Association of Diabetes Educators, 2014.

29. American Association of Diabetes Educators. Competencies for Diabetes Educators: A Companion Document to the Diabetes Educator Practice Levels Chicago: American Association of Diabetes Educators, 2014.

30. Marwick TH, Hordern MD, Miller T et al. Exercise training for type 2 diabetes mellitus - Impact on cardiovascular risk: A scientific statement from the American Heart Association. Circulation 2009; 119(25):3244-62.

31. Bhattarai MD. Three patterns of rising type 2 diabetes prevalence in the world: Need to widen the concept of prevention in individuals into control in the community. J Nepal Med Assoc. 2009;48(174):173-9.

32. Select Committee on Environment, Transport and Regional Affairs Eleventh Report. Walking in towns and cities. [online]. 2001 June 30 [cited 2015 June 3]; Available from: URL:http:/ / www.publications.parliament.uk/pa/cm200001/cmselect/ cmenvtra/167/16708.htm\#n

33. Beverage Marketing Corporation. University of Michigan Annual Consumer Satisfaction Index, Michigan, University of Michigan, 2005.

34. Chiasson JL, Josse RG, Gomis R, Hanefeld M, Karasik A, Laakso M, STOP-NIDDM Trail Research Group. Acarbose for prevention of type 2 diabetes mellitus: the STOP-NIDDM randomised trial. Lancet. 2002;359(9323):2072-7.

35. Rosenstock J, Brown A, Fischer J, Jain A, Littlejohn T, Nadaeu D, et al. Efficacy and safety of acarbose in metformin treated patient with type 2 diabetes. Diabetes Care 1998;21:2050-2055.

36. Halimi S, Le Berre MA, Grange V. Efficacy and safety of acarbose add-on therapy in the treatment of overweight patients with type 2 diabetes inadequately controlled with metformin: a double blind placebo-controlled study. Diabetes Research and Clinical Practice 2000;50:49-56. 
37. Jayaram S, Hariharan RS, Madhavan R, Periyandavar I, Samra SS. A Prospective, Parallel Group, Open-Labeled, Comparative, Multi-centric, Active Controlled Study to Evaluate the Safety, Tolerability and Benefits of Fixed Dose Combination of Acarbose and Metformin versus Metformin alone in Type 2 diabetes. J Assoc Physicians India 2010;48:679-687.

38. American Diabetes Association. Standards of medical care in diabetes 2016. Diabetes Care 2016;39(Suppl.1):S1-S111

39. Inzucchi SE, Bergenstal RM, Buse JB, et al.; American Diabetes Association (ADA); European Association for the Study of Diabetes (EASD). Management of hyperglycemia in type 2 diabetes: a patient centered approach: position statement of the American Diabetes Association (ADA) and the European Association for the Study of Diabetes (EASD). Diabetes Care 2012;35: 1364-1379

40. Cahn A, Raz I, Kleinman Y, et al. Clinical assessment of individualized glycemic goals in patients with type 2 diabetes: Formulation of an algorithm based on a survey among leading worldwide diabetologists. Diabetes Care 20152015 ;38:2293-2300.

41. Kearney PM, Whelton M, Reynolds K, Muntner P, Whelton PK, He J. Global burden of hypertension: analysis of worldwide data. Lancet. 2005;365(9455):217-23.

42. A policy statement from the American Heart Association. Impact of smokeless tobacco products on cardiovascular disease: Implications for policy, prevention, and treatment. Circulation 2010;122: 1-25.

43. Lindson-Hawley N, Banting M, West R, Michie S, Shinkins B, Aveyard P. Gradual Versus Abrupt Smoking Cessation: A Randomized, Controlled Noninferiority Trial. Ann Intern Med. 2016;164(9):585-92.
44. Cao Y, Willett WC, Rimm EB, Stampfer MJ, Giovannucci EL. Light to moderate intake of alcohol, drinking patterns, and risk of cancer: results from two prospective US cohort studies. BMJ 2015;351:h4238.

45. National Diabetes Education Program. Power to Prevent: A Family Lifestyle Approach to Diabetes Prevention. US: National Diabetes Education Program. (Available at: http:/ / ndep.nih.gov/media/power-to-prevent-508.pdf)

46. Board of Science. Board of Science Publications and Resources Timeline. London: British Medical Association (Available at: http://www.bma.org.uk/about-the-bma/ how-we-work/professional-activities-and-special-interest/ board-of-science/board-of-science-publications)

47. CancerCare. For Health Care Professionals. New York: CancerCare. (Available at: http://www.cancercare.org/ professionals)

48. British Heart Foundation. Professional Resources. London: British Heart Foundation. (Available at: https://www. bhf.org.uk/healthcare-professionals/resources-for-healthprofessionals)

49. CDC. Educational Materials for Professionals. Atlanta: CDC, 2015. (Available at: http://www.cdc.gov/heartdisease/ materials_for_professionals.htm)

50. WHO. Preparing a Health Care Workforce for the 21st Century - The Challenge of Chronic Conditions. Geneva: WHO, 2005.

51. Global Initiative for Asthma. Global Strategy for Asthma Management and Prevention. Global Initiative for Asthma, 2015

52. Global Initiative for Chronic Obstructive Lung Disease. Global Strategy for the Diagnosis, Management, and Prevention of Chronic Obstructive Pulmonary Disease. Global Initiative for Chronic Obstructive Lung Disease: 2014 